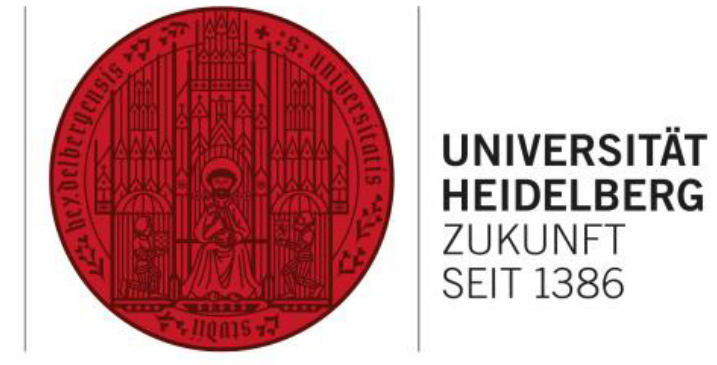

Zusammenfassung der Dissertation mit dem Titel

\title{
„Die internationale Steuerung der Biotechnologie am Beispiel des Umgangs mit neuen genetischen Analysen“"
}

Dissertation vorgelegt von Fruzsina Molnár-Gábor

Erstgutachter: Prof. Dr. Silja Vöneky

Zweitgutachter: Prof. Dr. Dr. h.c. Rüdiger Wolfrum

Juristische Fakultät 


\title{
Die internationale Steuerung der Biotechnologie am Beispiel des Umgangs mit neuen genetischen Analysen
}

\author{
Dissertation
}

Vorgelegt

\author{
an der Juristischen Fakultät der Ruprecht-Karls-Universität Heidelberg \\ von Fruzsina Molnár-Gábor
}

\section{Zusammenfassung}

Die Arbeit widmet sich dem internationalen Umgang mit den normativen Herausforderungen, die durch die modernen Entwicklungen der Biotechnologie hervorgerufen werden und untersucht diese am Beispiel neuer genetischer Analysen, insbesondere der vollständigen Sequenzierung des menschlichen Genoms. Ausgangspunkt und Ziel dieser Arbeit ist dabei, die Stellung des Patienten als Person zu beschreiben und aufrechtzuerhalten.

\section{Erster Teil: Die Stellung der Person}

Ziel des ersten Teils ist es, eine Vorstellung des normativen Umgangs mit der Person als Patient zu entwickeln, die - den medizinischen Schwerpunkt der Arbeit berücksichtigend als Basis für die weiteren Analysen dienen kann.

Im ersten Kapitel werden die wesentlichen Züge des Personenseins aufgrund philosophischgeistesgeschichtlicher Entwicklungslinien erläutert (Kapitel 1: Aspekte der Stellung der Person in der Geistesgeschichte). Die Ergbnisse des ersten Kapitels lassen sich wie folgt zusammenfassen.

Bereits im antiken Theaterwesen wurde eine gegenseitige Bedingtheit zwischen der Materialität und dem individuellen Charakter des Menschen im Begriff der Maske geschaffen. In der antiken Philosophie sind zudem einige der ersten Merkmale eines normativen Menschenbegriffs zu erkennen, die vor allem auf der Vernunftfähigkeit des Menschen basieren und sein regelgeleitetes Handeln erklären. Während die willentlichen Aspekte der Lebenswahl bei Epiktet eher als Verwirklichung der personellen Form des Lebens und der inneren Freiheit zu verstehen sind ${ }^{1}$, liegt der Schwerpunkt der Lebenswahl bei Cicero auf der

\footnotetext{
${ }^{1}$ M. Forschner, Der Begriff der Person in der Stoa, in: D. Sturma (Hrsg.), Person. Philosophiegeschichte Theoretische Philosophie - Praktische Philosophie, Mentis, Paderborn 2001, 37 ff., 42.
} 
Verwirklichung des Personenseins als Rollenträger in der Gesellschaft. ${ }^{2}$ Sehr früh wird der Mensch in den raumzeitlichen Kontext des gesellschaftlichen Zusammenlebens gesetzt. Dies passiert nicht nur aufgrund der Zuschreibung einer Rollenexistenz, sondern auch aufgrund der Sprachlichkeit.

In der christlichen Philosophie hatte sich das Verständnis der Metaphysik grundlegend verändert. Sie war nicht mehr eine theoretische, allgemeine Seinslehre oder eine abstrakte Gotteslehre, sondern eine Metaphysik des inneren Menschen. ${ }^{3}$ Die Eingliederung der Person in den Bereich des Moralischen erfolgte zu dieser Zeit. Bestimmender Wesenszug der Person wurde die Freiheit, die die der Person eigene Würde und ihren unendlichen Wert begründete. Vernunft und freier Wille als Spezifika menschlicher Natur begründen auch bei Pufendorf das sittliche Handeln und die daraus ableitbare Würde des Menschen. Aufgrund dieses Status kommen dem Menschen unveräußerliche Rechte und Pflichten zu. $^{4}$ Die Fähigkeiten der Vernunft und des freien Willens bieten dem Menschen aber nur in der Gemeinschaft ein Betätigungsfeld. ${ }^{5}$ Der Gedanke Pufendorfs über unveräußerliche Rechte, aber auch Pflichten der „Menschheit““ wird in den Vertragstheorien der Neuzeit weiterentwickelt.

In der Neuzeit entstand ein Verständnis von dem der Freiheit eigenen metaphysischen Charakter: Der Mensch wird als Wesen der Freiheit aufgefasst, in der seine Personalität begründet liegt. Kant betont, dass Personalität sich im Handeln zeigt und deshalb für die Ethik relevant ist. ${ }^{6}$ Er versteht Personalität als Freiheit und Unabhängigkeit von bloßer Naturkausalität. ${ }^{7}$ Der in der Freiheit bedingte absolute Wert ist unmittelbar gegeben. Autonomie, die Personen erlaubt, Zwecke zu setzen und sich durch Gründe bestimmen zu lassen, bewirkt, dass Menschen als Selbstzweck existieren und nicht instrumentalisiert werden dürfen, sondern auch als Wesen begriffen werden müssen, die schuld- und verantwortungsfähig sind. ${ }^{8}$ Eine „sozialphilosophische Korrektur“ von Kants Theorie wurde von Fichte und Hegel vorgenommen. ${ }^{9}$

\footnotetext{
${ }^{2}$ M. Fuhrmann, Persona, ein römischer Rollenbegriff, in: O. Marquard/K. Stierle (Hrsg.), Identität. Poetik und Hermeneutik, Bd. VIII, Wilhelm Fink, München 2. Aufl. 1996, 83 ff., 87 ff., 101.

${ }^{3}$ T. Kobusch, Die Entdeckung der Person. Metaphysik der Freiheit und modernes Menschenbild, Herder, Freiburg 1993, 139.

${ }^{4}$ Id., 75 .

${ }^{5}$ R. Wolfrum, Samuel Pufendorfs Blick auf die EU. Eine Hypothese, in: Ch. Baldus/H. Kronke/U. Mager (Hrsg.), Heidelberger Thesen zu Recht und Gerechtigkeit, Mohr Siebeck, Tübingen 2013, 27 ff., 34 ff.

${ }^{6}$ R. Kather, Person. Die Begründung menschlicher Identität, Wissenschaftliche Buchgesellschaft, Darmstadt 2007, 9 .

${ }_{7}$ D. Sturma, Person, in: H. J. Sandkühler (Hrsg.), Enzyklopädie Philosophie, Bd. 2, Felix Meiner Verlag, Hamburg 2010, 1921 ff., $1922 \mathrm{f}$.

${ }^{8}$ I. Kant, Grundlegung zur Metaphysik der Sitten, Kommentar von C. Horn/C. Mieth/N. Scarano, Suhrkamp, Frankfurt am Main 2007, AA IV 428, 8.

${ }^{9}$ Sturma (Fn. 7), 1923.
} 
In der neuzeitlichen ethischen Diskussion des Personenbegriffs wurde die Materialität zugunsten der Bestimmungen der Vernunft, der Freiheit und des Selbstbewusstseins einer Person in den Hintergrund gestellt. Die Begründung des sittlichen Charakters der Person und die Zuschreibung von Würde und Verantwortung werden insbesondere bei Kant und Locke durch Bestimmungen dieser Eigenschaften vorgenommen. Die im 20. Jahrhundert entwickelten ethischen Personentheorien wurden durch ihre Thesen stark beeinflusst.

Erst in der Phänomenologie ergänzt die Leiblichkeit den auf Vernunft, Selbstbewusstsein und Autonomie basierenden moralischen Personenbegriff explizit. Der Leib als personaler Körper verbindet die geistigen Elemente mit der Raumzeitlichkeit und Intersubjektivität, wobei die normativen Personenbestimmungen, Vernunft und Autonomie, aufrechterhalten bleiben. Die Phänomenologie erachtet diese Verbindung als geeignet, die normativen Funktionen, die der Person zugesprochen werden, $\mathrm{zu}$ erfüllen. ${ }^{10}$ Lévinas verbindet Antisolipsismus und Leiblichkeit systematisch und fundiert Freiheit und Selbstbewusstsein der Person in einer ethischen Beziehung zu anderen. ${ }^{11}$

Die Begründung der Anerkennung und Eingebundenheit der Person in den Kontext von Mitmenschen und Gesellschaft wird sodann im 21. Jahrhundert vor allem von der modernen Soziologie und der politischen Philosophie geleistet. Plessner greift auf den antiken Begriff der Maske zurück und nutzt ihn, um eine Soziologie gegen die Entfremdung des Menschen zu entwickeln. $^{12}$ Margalit positioniert den Menschen in der ihn aufgrund seiner Würde anerkennenden und schützenden Gesellschaft. ${ }^{13}$

Die weitere Analyse basiert auf die Stellung der Person in der Geistesgeschichte unter Berücksichtigung der im ersten Kapitel hervorgehobenen, sie in ihrer Ganzheitlichkeit beschreibenden Dimensionen. Diese muss als eine mehrdimensionale Stellung wahrgenommen werden, die sowohl die Leiblichkeit und Verletzlichkeit der Person als auch eine dynamische Normativität umfasst. Diese Normativität ist in der Vernunfthaftigkeit angelegt, beachtet die sich in der Freiheit zeigende Individualität und Einmaligkeit und lässt die Bezüglichkeit zur Mitwelt im Sinne von sozialen Strukturen, kulturellen Kontexten und Anerkennungsverhältnissen zu.

\footnotetext{
10 A. Reichold, Die vergessene Leiblichkeit. Zur Rolle des Körpers in ontologischen und ethischen Personentheorien, Mentis, Paderborn 2004, 224 f., 228 f.

${ }^{11}$ Id., $193 \mathrm{f}$.

${ }^{12}$ Kobusch (Fn. 3), 246 f.

${ }^{13}$ A. Thumfart, Die Würde des Menschen: Giovanni Pico della Mirandola, Albrecht Dürer und Avishai Margalit, 51 Zeitschrift für Politik 4 (2004) 434 ff., 454.
} 
Im zweiten Kapitel wird die Stellung der Person in der überstaatlichen Rechtsordnung skizziert (Kapitel 2: Die Stellung der Person in der internationalen Rechtsordnung). Es wird untersucht, welche Aspekte einer universalistischen Stellung der Person als normatives und raumzeitliches Wesen in der geltenden internationalen Rechtsordnung aufgefangen werden.

Die Aufschlüsselung der Stellung der Person im Recht kann im Wesentlichen aufgrund der ihr zugesprochenen Würde und Rechte erfolgen. Als moralisches, leibliches und soziales Wesen ist der Mensch Subjekt der Menschenwürde und der Menschenrechte. Als selbstbestimmte Person, als zur Sittlichkeit fähige Persönlichkeit wird ihm Würde und Freiheit zugesprochen, Gleichheit vor dem Gesetz und ein soziales Willkommen in der Rechtsgemeinschaft zugesichert. ${ }^{14}$ Trotz der Begründung der Würde in sittlich-geistigen Eigenschaften von Vernunft und Gewissen wird die Person als Einheit von Geist und Körper interpretiert. Der Ausgangspunkt der Stellung der Person, die Menschenwürde, kann nicht losgelöst von einer konkreten Anerkennungsgemeinschaft, in der die Person auch als leibliches Wesen präsent ist, gedacht werden. In den reziproken Anerkennungsbeziehungen kommunikativ handelnder Personen wird der Mensch lernen, sich selbst als einzigartiges, zugleich als moralisch unvertretbares Individuum zu identifizieren - ihm kommen als Teil der Gemeinschaft Rechte zu. $^{15}$

Diese Auslegung wird Grund und Grenze der internationalen Rechtsordnung. ${ }^{16}$ Die Stellung der Person im internationalen Menschenrechtsregime entspricht demnach einem metaphysischen und relationalen Charakter und einem, dessen Aufgabe mit dem Anspruch auf die Begrenzung staatlicher Macht verbunden ist. ${ }^{17}$

Die Stellung der Person als normativ-raumzeitliches Wesen kann für bestimmte Kontexte konkretisiert werden, indem ausgewählte Aspekte dieser Stellung einem besonderen Schutz unterzogen werden. Im dritten Kapitel wird untersucht, welche Aspekte in der medizinischen

\footnotetext{
${ }^{14}$ P. Kirchhof, Menschenbild und Freiheitsrecht, in: R. Grote/I. Härtel/K.-E. Hain et al. (Hrsg.), Die Ordnung der Freiheit. FS für Christian Starck zum 70. Geburtstag, Mohr Siebeck, Tübingen 2007, 275 ff., 276.

15 J. Habermas, Die Zukunft der menschlichen Natur. Auf dem Weg zu einer liberalen Eugenik? Suhrkamp, Frankfurt am Main, 2001, 66. Diese Auslegung spricht auch gegen eine Definition des Menschen durch ,das Gesetz“. Vgl. W. Höfling, Definiert die Rechtsordnung den Menschen? In: W. Schweidler (Hrsg.), Menschenleben - Menschenwürde. Internationales Symposium zur Bioethik, Lit Verlag, Münster/Hamburg/London 2003, 165 ff., 166 ff.

${ }^{16}$ Wie Matthias Mahlmann zutreffend feststellt, verkörpert dies den Inbegriff einer aufgeklärten, humanistischen Ethik der gleichberechtigten Selbstzweckhaftigkeit aller Menschen. Eine weltanschauliche, philosophische Neutralität der Würdekonzeption ist demnach insoweit nicht zu haben. M. Mahlmann, Elemente einer ethischen Grundrechtstheorie, Nomos, Baden-Baden 2008, $351 \mathrm{f}$.

${ }^{17}$ In Anlehnung an Ch. McCrudden, Human Dignity and Judicial Interpretation of Human Rights, 19 European Journal of International Law 4 (2008) 655 ff., $679 \mathrm{f}$.
} 
Ethik und in Anbetracht der Patientenrechte wichtig sind, um eine gemeinsame normative Vorstellung über den Umgang mit Patienten als Personen nachzuvollziehen (Kapitel 3: Die Stellung der Person als Patient).

Betrachtet man den Umgang mit Patienten im Spiegel ethischer und rechtlicher Maßstäbe, so wird zuerst das Verhältnis zwischen Ethik und Recht im Allgemeinen und zwischen medizinischer Ethik, Bioethik und den Patientenrechten im Besonderen geklärt. Diese Klärung ergibt, dass sowohl die Ethik, insbesondere die medizinische Ethik, als auch das Recht, insbesondere in seiner Manifestierung im Menschenrechtssystem, wesentlich und eigenständig zur Konkretisierung der Stellung der Person als Patient beitragen können. Eine gewisse Überlappung, den gemeinsamen Ursprüngen und Zielen geschuldet, ist dennoch nicht zu verhindern. Eine Inkorporierung ethischer Prinzipien in die rechtlichen Regelungen kann für den Umgang mit Patienten allerdings fruchtbar sein.

Vor allem im Arzt-Patienten-Verhältnis, das als eine partnerschaftliche Beziehung aufgefasst wird, wird die Stellung des Patienten als raumzeitliches aber auch normatives Wesen in der konkreten Situation einer Krankheit gewährleistet. Er wird in seiner Lebensgeschichte mit Rücksicht auf seine Überzeugungen, Ängste und Wünsche behandelt.

Menschenrechte schützen den Menschen auch im Bereich der Medizin: Der Person als Patient kommen die Menschenrechte auch in medizinischen Kontexten zu.

In der Medizin ist die Person in erster Linie als verletzbares und sterbliches Wesen präsent. Ihre körperliche Verfasstheit qualifiziert sie überhaupt als Gestalt, die krank werden kann und ärztliche Hilfe braucht. Sie darf aber nicht als nur bloßes Objekt medizinischer Eingriffe behandelt werden, sondern muss die Funktion eines Subjekts der Verantwortung immer in einem Verhältnis zu sich und zu anderen Personen durch ihre Stellung erfüllen können. Die Voraussetzung hierfür wird dadurch gewährleistet, dass dem Individuum die Kontingenz, die natürliche Unfestgestelltheit seiner Körperlichkeit als Moment seiner ethischen Individualität auch in der besonderen Lebenssituation der Krankheit nicht genommen wird. ${ }^{18}$

Die dynamische Interpretation und Konkretisierung der Menschenrechte vor allem in der internationalen Gerichtsbarkeit führen dazu, dass sie der technischen Entwicklung in der Medizin und den sich daraus ergebenden gesellschaftlichen Änderungen Rechnung tragen können. ${ }^{19}$ Die Einordnung der Stellung des Patienten im System der Menschenrechte ist

\footnotetext{
18 H. Hofmann, Biotechnik, Gentherapie, Genmanipulation - Wissenschaft im rechtsfreien Raum? 41 JuristenZeitung 6 (1986) 253 ff., 260.

${ }^{19}$ Für eine spezielle Konvention plädierend aber J.-P. Jacqué, Europäische Menschenrechtskonvention und Bioethik, in: F. Furkel/H. Jung (Hrsg.), Bioethik und Menschenrechte/Bioéthique et droits de l'homme, Heymann, Köln u. a. 1993, 1 ff., 9.
} 
angesichts neuer Herausforderungen daher nicht abgeschlossen. Sie kann und soll Gegenstand weiterer Entwicklungen werden.

\section{Zweiter Teil: Die Herausforderung der medizinischen Entwicklung am Beispiel der Gesamtgenomsequenzierung}

Im zweiten Teil der Arbeit werden die Herausforderungen für eine Stellung des Patienten, wie in Teil 1 bestimmt, unter dem Einfluss der naturwissenschaftlich-medizinischen Entwicklung mit Blick auf die neuen Untersuchungsmöglichkeiten des menschlichen Genoms analysiert. Um diese Herausforderungen $\mathrm{zu}$ identifizieren, bedarf es zuerst einer Darstellung der humangenetischen Entwicklung in den letzten Jahrzehnten. Danach folgt eine technologische Beschreibung des aktuellen Potenzials genomweiter Analysen, insbesondere der Genomsequenzierung (Kapitel 4: Die Genomsequenzierung. Eine technologische Analyse). Hierzu lässt sich festhalten, dass die Sequenzierung großer genomischer Bereiche oder ganzer Genome mit hoher diagnostischer Genauigkeit es erlaubt, komplexe Mutationsspektren zum überschaubaren Kostenaufwand zu analysieren. Die Aufklärung kausaler Zusammenhänge zwischen Genveränderungen und komplexen Stoffwechselwegen wird in absehbarer Zeit im Hinblick auf genetisch bedingte Krankheiten möglich. Das technische Potenzial, die Informationen auszuwerten, wird ständig erweitert. Bei einer Untersuchung, der die Isolierung der gesamten genomischen DNA vorausgegangen ist, können daher das Gesamtgenom ausgelesen und bereits heute viele Veränderungen identifiziert und validiert werden.

Die Einführung der Genomsequenzierung in die Medizin beeinflusst die Stellung des Patienten, welche in den besonderen Eigenschaften dieser Technologie begründet ist (Kapitel

\section{5: Die Genomsequenzierung. Eine normative Analyse).}

Die umfassende Analyse des menschlichen Genoms durch eine Sequenzierung stellt in medizinischen Kontexten zunächst einen Informationseingriff dar. ${ }^{20}$ Bei dieser neuartigen Vorgehensweise der genetischen Untersuchung ist nicht der Eingriff in die körperliche Unversehrtheit, sondern die Beschaffung von Daten Gegenstand der Einwilligung. Das qualitativ Neue der vollständigen Genomsequenzierung besteht darin, dass durch die totale Entschlüsselung des Genoms eine äußerst hohe Anzahl an genetischen Veränderungen und Befunden entdeckt wird. Dies bedeutet, dass eine nicht $\mathrm{zu}$ überblickende Menge an

\footnotetext{
${ }^{20}$ F. Molnár-Gábor/J. Weiland, Die Totalsequenzierung des menschlichen Genoms als medizinischer Eingriff Bewertung und Konsequenzen, 60 Zeitschrift für medizinische Ethik 2 (2014) 135 ff.
} 
genetischem Informationen generiert wird oder zumindest generiert werden kann, die Aufschluss über verschiedenste Anlageträgerschaften geben, aber gleichzeitig im diagnostischen Kontext regelmäßig keine Aussage über die medizinische Ausgangsfrage des Patienten zulassen. Einerseits öffnet die enorme Vielzahl an zu erwartenden Veränderungen und Befunden die Tür für prädiktive Diagnostikmöglichkeiten. ${ }^{21}$ Andererseits ist der größte Teil der durch eine Gesamtgenomsequenzierung erkennbaren Sachverhalte im Voraus nicht zu spezifizieren und gleichzeitig so zahlreich sowie ihre Interpretation an medizinischgenetische Fachexpertise so stark gebunden, dass das Wissen des behandelnden Arztes und der Verständnishorizont eines Patienten weit überschritten werden. Manche Veränderungen und Befunde können aber auch nach der Einholung molekularbiologischer Expertise nur mit einer gewissen Durchschlagskraft, d.h. als Risikoinformationen beziffert werden. Die Einordnung ihrer gesundheitlichen Relevanz bleibt letztlich damit dem subjektiven Beurteilungsspielraum des Patienten überlassen.

Eine weitere Schwierigkeit stellt die Tatsache dar, dass die wissenschaftliche Beurteilung der Befunde teilweise noch nicht abschließend vorgenommen werden kann. ${ }^{22}$ Darüber hinaus können sich durch die Entwicklung der Forschung Untersuchungsmaßstäbe verändern und zu weiteren Informationen führen. ${ }^{23}$ Insbesondere aufgrund des wissenschaftlichen Fortschritts können aus dem sequenzierten und gespeicherten Genom mit der Zeit weitere Informationen gewonnen werden. Aufgrund der ständig neuen Informationsgewinnung stellt sich die Analyse des Genoms nicht als ein punktueller Eingriff, sondern als ein dynamischer Prozess dar, durch den auch in die Rechte des Betroffenen sukzessiv und andauernd eingegriffen wird. $^{24}$ Der informationelle Eingriff im Rahmen einer Gesamtgenomanalyse ist zudem so umfassend, dass er auch die genetisch Verwandten betrifft.

Bei der Einführung der Gesamtgenomanalyse in die medizinische Diagnostik setzen international aufgestellte Forschungsprojekte und Forschungsorganisationen die Maßstäbe. Zwar handelt es sich zunächst bei den Projekten um Forschungsprojekte, ihr Beitrag zur Implementierung der Genomsequenzierung in der medizinischen Diagnostik geht aber über die der einfachen Forschung hinaus. Dies ist damit $\mathrm{zu}$ begründen, dass die meisten

\footnotetext{
${ }^{21}$ F. Molnár-Gábor/G. Gantner/P. Lichter, Das Ende der Zufallsbefunde in der genetischen Diagnostik. Die Ganzgenomsequenzierung und die Erwartbarkeit zusätzlicher Befunde, Zeitschrift für Medizin-Ethik-Recht 2 (2014) $81 \mathrm{ff}$.

${ }^{22}$ D. Gadzicki, Der Stand des genetischen Möglichen und die Rolle der genetischen Beratung - jetzt und in Zukunft, in: Th. Moos.JJ. Niewöhner/K. Tanner (Hrsg.), Genetisches Wissen, Röhrig Universitätsverlag, St. Ingebert 2011, 49 ff.

${ }^{23}$ S. M. Liao, Is there a duty to share genetic information? 35 Journal of Medical Ethics 4 (2009) $306 \mathrm{ff}$.

${ }^{24}$ Molnár-Gábor/Weiland (Fn. 20), 138 ff.
} 
Forschungsvorhaben einen translationalen Charakter haben. Demnach fließen die Forschungsergebnisse möglichst unmittelbar in die medizinische Behandlung und Therapie der Patienten ein, die an bestimmten klinischen Studien oder Projekten teilnehmen. Die Zusammenarbeit zwischen Institutionen dieser translationalen Forschung erfolgt zunehmend multizentral und grenzüberschreitend.

Im Ergebnis bedeutet die Anwendung einer Gesamtgenomanalyse eine besondere Herausforderung für die Stellung des Patienten als Person auf drei Ebenen: Bei der Stellung des Patienten zu sich selbst, bei seinem Verhältnis zum Arzt und in Bezug auf seine Rechte.

(Kapitel 6: Die Konsequenzen der Anwendung der Gesamtgenomanalyse in der translationalen Medzin für Ihre Steuereung).

Die Gesamtgenomanalyse führt zu einer Technisierung und zunehmender Objektivierung von Gesundheits- und Krankheitschancen, die schließlich in einer Selbstverobjektivierung des Betroffenen resultieren kann. ${ }^{25}$ Das Kranksein entscheidet sich auf der Ebene des Genoms und wird mit Hilfe von Sequenziergeräten festgestellt. Die Speicherung der Sequenz des Betroffenen ermöglicht die Weiterführung der Analysen über eine erste und einmalige Untersuchung hinaus. Der Patient verschwindet als leiblich präsentes Wesen aus der Beziehung zum Arzt, er muss als solches für die fortdauernde Auslesung des Genoms nicht anwesend sein. Es besteht die Gefahr, dass der Patient auf die Menge der Daten aus seinem Genom reduziert wird.

Nicht nur der Patient verschwindet aus der Arzt-Patienten-Beziehung. Auch der Arzt wird in seinen ursprünglichen Funktionen kaum mehr gebraucht. Die Interpretation und Auslesung der genetischen Informationen bedarf molekularbiologischer Expertise, über die er nicht verfügt. Ist die Information einmal entschlüsselt, kann der Arzt sie dem Patienten meistens nur als Risikoinformation weitergeben und somit keine herkömmlichen, therapeutisch indizierten Maßnahmen dazu anordnen oder durchführen. Die weitere Interpretation und der Umgang mit Risikoinformationen wird Aufgabe des Patienten. Er wird auch zunehmend in

\footnotetext{
${ }^{25}$ C. G. van El/ M. C. Cornel/P. Borry et. al., Whole-genome sequencing in health care, 21 European Journal of Human Genetics 6 (2013) 580 ff., 581. Siehe das Fallbeispiel D. Winkler/Ph. Lyrer, Prevention of Harmful False Diagnosis versus Threat by Disclosure of an Inheritable Disease - A Medical Case, in: Ch. Rehmann-Sutter/H. Müller (Hrsg.), Disclosure Dilemmas. Ethics of Genetic Prognosis after the 'Right to know/Not to Know' Debate, Ashgate, Farnhma/Burlington 2009, 39 ff., 42. Siehe auch den Kommentar zu dem Fall von Christoph RehmannSutter, Communication Yes, but How - and What? In: Ders./Müller, id., 45 ff., 48 f. Auch die Entscheidungsgeschichten zweier Patienten schildern die Selbstobjektivierungsgefahr: R. Porz, The Need for an Ethics of Kinship: Decision Stories and Patients's Context, in: Rehmann-Sutter/Müller, id., 53 ff., 55 ff. Für die normative Analyse dieser Fragen siehe nur T. Tymstra, 'If I Only Had (Not) Known That': About RiskIndividualization, Chance Specification and the Loss of Certainty of Not-Knowing, in: Rehmann-Sutter/Müller, id., $85 \mathrm{ff}$.
} 
die Verantwortung genommen; nicht nur die Interpretation und der Umgang mit den Risikoinformationen werden seinem subjektiven Ermessen überlassen, sondern ihm wird eine gesteigerte Verantwortung, den Ausbruch potenzieller Krankheiten zu verhindern, auferlegt. ${ }^{26}$ Es droht ein Übergang zu einer Gesundheitspflicht, die die Selbstbestimmung als Freiheit zur Krankheit erübrigt. $^{27}$

Die Steuerung der Gesamtgenomanalyse wird dann Anerkennung finden, wenn sie kontextsensitiv vonstattengeht, das heißt die Eigenschaften der Gesamtgenomanalyse berücksichtigt und die mit ihrer Anwendung einhergehenden Herausforderungen beantwortet. Aufgrund ihrer Besonderheiten fordert die Anwendung dieser Technologie spezielle normative Lösungen für die Aufrechterhaltung und Konkretisierung einer ,personenhaften“ Stellung des Patienten als moralisch-raumzeitlicher Akteur in der Medizin. Bei ihrer Steuerung soll daher insbesondere auf das etablierte Personenverständnis zurückgegriffen werden; eine Steuerung, die sich an einem solchen Verständnis der Person orientiert, wird notwendig. ${ }^{28}$

Die Berücksichtigung der Eigenschaften der Gesamtgenomanalyse und die Lösung der mit ihrer Anwendung einhergehenden Herausforderungen werden daher im größeren analytischen Rahmen der Anerkennungswürdigkeit ihrer Steuerung auf internationaler Ebene de lege lata und de lege ferenda im dritten Teil der Arbeit betrachtet. Vor der Betrachtung dieser Fragen wird der Rahmen für die Analyse festgelegt.

Hierbei wird auf die völkerrechtliche Legitimität als Voraussetzung und Grenze für die Anerkennungswürdigkeit einer internationalen Steuerung der Gesamtgenomanalyse abgestellt. Obwohl keine einheitliche Theorie für die Definition der völkerrechtlichen Legitimität und für die Bestimmung ihrer Voraussetzungen existiert, gibt es bei den verschiedenen Interpretationen gemeinsame Ansätze. Diese können als Faktoren identifiziert werden, die zwar auf unterschiedliche Art und Weise, aber über viele Theorien hinweg einen Einfluss auf die Legitimität des Völkerrechts haben. Demnach sind die Legitimität internationaler Regelungen herbeiführende oder beeinflussende Faktoren solche, die mit der

\footnotetext{
${ }^{26}$ R. Kollek/T. Lemke, Der medizinische Blick in die Zukunft. Gesellschaftliche Implikationen prädiktiver Gentests, Campus, Frankfurt am Main/New York 2008, $223 \mathrm{ff.}$

${ }^{27}$ G. Duttge, ,Was meint genetische Individualität?“ - Eine Problemskizze, in: G. Duttge/W. Engel/B. Zoll (Hrsg.), Genetische Individualität im Recht, Göttinger Schriften zum Medizinrecht, Bd. 13, Universitätsverlag Göttingen, Göttingen 2012, 3 ff., 5 f.

${ }^{28}$ Für die philosophische Definition der Person als die Möglichkeit eines ethisch-raumzeitlichen Wesens siehe D. Sturma, Person, in: P. Kolmer/A. G. Wildfeuer (Hrsg.), Neues Handbuch philosophischer Grundbegriffe, Bd. II, Verlag Karl Alber, Freiburg im Breisgau 2011, 1728 ff., 1735 f. (7).
} 
Quelle ihrer Entstehung, mit dem Verfahren und der Transparenz der Entscheidungsfindung und nicht zuletzt mit den erzielten Ergebnissen verbunden sind. ${ }^{29}$

Die Bemühung um eine Stellung des Patienten als Person trägt wesentlich zu der normativen und faktischen Anerkennungswürdigkeit der internationalen Steuerung genetischer Analysen bei. Die auf diese Weise entstehende Anerkennungswürdigkeit der Steuerung, die vom thematischen Gegenstand der Regulierung abhängt, steht im engen Zusammenhang mit rechtstheoretischen Faktoren, die die Legitimität der Steuerung beeinflussen oder herbeiführen können. Demnach ist der an der Person orientierte Umgang mit der Ganzgenomanalyse nicht von Faktoren einer rechtstheoretischen Legitimation der internationalen Steuerung zu trennen.

Die Kriterien der Analyse richten sich damit nach den rechtstheoretisch anerkannten Faktoren, die einen Einfluss auf die Legitimität internationaler Steuerung ausüben können. Es werden die Fragen gestellt, welches Mandat für die Steuerung im Bereich der Ethik und des Rechts vorhanden ist, was die Quelle der Entstehung der Regelungen zur Genanalyse angesichts eines Auftrags und der thematischen Ansätze ist, und wie das Mandat in Bezug auf die Normativität und die Verbindlichkeit der Steuerung umgesetzt wird. Der Frage nach der Normativität der Steuerung geht die Frage voran, in welchen Verhandlungsprozessen die Regelungen zustande kommen: Wer nimmt an diesen Prozessen teil und wie werden die Regelungen inhaltlich ausgearbeitet? Das Ergebnis der Steuerung soll sowohl angesichts der Effektivität ihrer Inhalte als auch der Förderung und Umsetzung der Regelungen analysiert werden.

Bevor diese Analyse angegangen werden kann, wird zuerst klargestellt, welcher institutionellen Einbindung die internationale Steuerung der Genanalyse unterliegt.

Vor dem Hintergrund der grenzüberschreitenden Herausforderungen der Patientenstellung und der grenzüberschreitenden Zusammenarbeit in der translationalen Medizin stellen die Vereinten Nationen und ihre Sonderorganisationen für die Analyse einen geeigneten Gegenstand dar. An erster Stelle bei der Entwicklung internationaler biomedizinischer Standards steht die Organisation der Vereinten Nationen für Erziehung, Wissenschaft und Kultur (UNESCO). Die Zuständigkeit für die Befassung mit biomedizinischen Fragen leitet die UNESCO aus der Präambel ihrer Satzung ab. ${ }^{30}$

\footnotetext{
${ }^{29}$ Vgl. $R$. Wolfrum, Legitimacy in International Law, in: Ders. (Hrsg.), MPEPIL, März 2011, Rn. 5 ff.; F. Muñoz Hernandez, Legitimität und Legalität im Völkerrecht. Die völkerrechtstheoretische Debatte um die Legitimität Governance-Strukturen, Inaugural-Dissertation Universität Tübingen 2011, 48 ff.

${ }^{30}$ UNESCO, Constitution of the UNESCO, 4 UNTS 275, UN Reg No I-52.
} 


\section{Dritter Teil: Steuerung der Gesamtgenomanalyse im Grenzbereich von Ethik und Recht}

\section{de lege lata und de lege ferenda}

Übergeordnetes Ziel dieses Teils ist es aufzuzeigen, welchen Einfluss die Berücksichtigung der Eigenschaften und Herausforderungen der Gesamtgenomanalyse bei der Steuerung genetischer Analysen auf die Faktoren ihrer Anerkennungswürdigkeit de lege lata ausgeübt hat und de lege ferenda ausüben kann.

Zunächst gilt es zu untersuchen, ob die auf internationaler Ebene bestehenden spezifischen Regelungen anerkennungswürdige Antworten auf die Problemverschärfung durch eine Anwendung der Gesamtgenomanalyse als medizinischer Eingriff in der Arbeit der UNESCO bereithalten. (Kapitel 7: Genetische Analysen im internationalen Menschenrechtssystem auf universeller Ebene - de lege lata).

Auf internationaler Ebene wurde früh erkannt, dass die rasante Entwicklung der Genetik große Gefahren aber auch ein großes Therapiepotenzial birgt. Der Beitrag der UNESCO zur Förderung der Ethik, insbesondere der biomedizinischen Ethik, wird bei der Standardsetzung bisher vor allem in Form von unverbindlichen Erklärungen geleistet. Bisher wurden drei Erklärungen verabschiedet. Die ersten beiden, die Allgemeine Erklärung über das menschliche Genom und Menschenrechte $1997^{31}$ und die Internationale Erklärung über humangenetische Daten $2003^{32}$, behandeln den engeren Themenbereich der Humangenetik. Die dritte, die Allgemeine Erklärung über Menschenrechte und Bioethik 2005, beschäftigt sich mit übergreifenden ethischen Fragestellungen der Medizin, der Lebenswissenschaften und den dazugehörigen Technologien. ${ }^{33}$

Die vergleichende Analyse der biomedizinischen Steuerung durch die UNESCODeklarationen ergibt folgende Resultate.

Alle drei Deklarationen wurden im Rahmen des ethischen Mandats und entsprechend der Schlüsselaktivität der UNESCO innerhalb des Mandats erarbeitet und gelten als unverbindliche Instrumente für die Steuerung biomedizinischer Fragestellungen. Sie wurden alle mit Bezug auf die Menschenwürde verabschiedet und in den verrechtlichten menschenrechtlichen Referenzrahmen eingegliedert.

\footnotetext{
${ }^{31}$ Universal Declaration on the Human Genome and Human Rights v. 11.11.1997, Records of the General Conference, $29^{\text {th }}$ Session, Paris, 21.10.-12.11.1997, Bd. 1, 41 ff., 29 C/Resolution 16.

${ }^{32}$ International Declaration on Human Genetic Data v. 16.10.2003, UNESCO-General Conference, $32^{\text {nd }}$ Session, Paris, 29.9.-17.10.2003, Bd. 1, 39 ff., 32 C/Resolution 22.

33 Universal Declaration on Bioethics and Human Rights v. 19.10.2005, Records of the UNESCO-General Conference, $33^{\text {rd }}$ Session, Paris, 3.-21.10.2005, Bd. 1, 74 ff., 33 C/Resolution 36.
} 
Die Aufträge für die Erarbeitung dieser Instrumente wurden von der Generalkonferenz an den Generaldirektor erteilt. Thematische Ansätze wurden im Vorfeld der Erarbeitung neben den Aufträgen vor allem in den Berichten des IBC angerissen, die jeweils von hoher Sensibilität bei der Betrachtung biomedizinischer und technologischer Herausforderungen zeugen. Seinen Berichten, die zur Vorbereitung der verabschiedeten Deklarationen dienten, liegt mittelbar auch das Ziel zugrunde, den Menschen in seiner Personenhaftigkeit als ethisch-leibliches Wesen, vor allem in der Manifestation dieser in der Menschenwürde und den Menschenrechten aufrechtzuerhalten.

In Bezug auf den Ausarbeitungsprozess können folgende Merkmale unterstrichen werden. Über die Beteiligung am Ausarbeitungsprozess lässt sich zuerst generell festhalten, dass die Einbindung der Entwicklungsländer in die Erarbeitung der Deklarationen auf allen Ebenen gewährleistet wurde. Dies zeichnet die Entscheidungsfindung der UNESCO insbesondere im Vergleich zu der Revision der Helsinki-Deklaration des Weltärztebundes und der Richtlinien des Rats für Internationale Organisationen der medizinischen Wissenschaft [CIOMS] über internationale biomedizinische Forschung aus, da letztere fast ausschließlich von westlichen Wissenschaftlern erarbeitet wurden und die Meinungen der Entwicklungsländer erst rückwirkend oder zu wenig eingebunden wurden. ${ }^{34}$ Obwohl die Entwicklungsländer mit ihren Repräsentationsmöglichkeiten und mit der Wahrnehmung ihrer Vorschläge generell eher unzufrieden waren, zeigten sie sich mit den Ergebnissen der Verhandlungen grundsätzlich zufrieden. $^{35}$

Die Zusammensetzung des International Bioethics Committee [IBC] selbst hat sich im Laufe der Jahre zunehmend interdisziplinarisiert. Während die Besetzung bei der Erarbeitung der ersten Deklaration noch von Juristen dominiert wurde, stieg bei den letzten beiden Deklarationen die Anzahl der beteiligten Mediziner und der Naturwissenschaftler und bei der letzten Deklaration auch die Anzahl der Philosophen insgesamt. Zudem ist die Tendenz der Einbindung einer zunehmenden Zahl von Mitgliedern mit Erfahrung in nationalen Ethikkommissionen zu beobachten. Die Beibehaltung der Mitglieder, die auch in der Human Genome Organization engagiert waren, machte die Einbringung spezifischer genetischer Fragestellungen in die Arbeit möglich.

\footnotetext{
${ }^{34}$ U. Schücklenk/R. Ashcroft, International Research Ethics, 14 Bioethics 2 (2000) 158 ff., 170.

${ }^{35}$ A. Langlois, Negotiating Bioethics, Routledge, London 2013, 144 ff.
} 
Zwar gibt es keinen internationalen Verhandlungsprozess, der ausschließlich technischen Experten der Regierungen überlassen werden kann, ${ }^{36}$ die Einbindung der Politik in die Erarbeitung der Deklarationen geschah aber auffällig früh bereits auf der Ebene des IBC, denn in allen Zusammensetzungen war ein bedeutender Teil seiner Mitglieder in den Mitgliedstaaten auch staatlich-politisch oder diplomatisch engagiert. Die Finalisierung der Deklarationen wurde jeweils von den Repräsentanten der Mitgliedstaaten in den Intergovernmental Bioethics Committee [IGBC] vorgenommen und die zu verabschiedende Fassung in den sogenannten intergouvernementalen Expertentreffen [IGE-Treffen] erstellt. Dies bedeutet, dass die Staatenrepräsentanten und nicht das Ethikkomitee die finale Fassung der Deklarationen zur Annahme durch die Generalkonferenz verabschiedet hat. Sowohl der IGBC als auch die jeweils zwei finalisierenden Treffen der Staatenrepräsentanten haben mehr als nur den letzten Feinschliff oder rein formelle und strukturelle Änderungen vorgenommen, zumal Entscheidungen über Fragen, über die im Laufe des Erarbeitungsprozesses keine Einigung erzielt werden konnte, mehrfach während dieser letzten Treffen geklärt wurden.

Dies gab bei allen drei Deklarationen Anlass zu der Kritik, dass die ethische und wissenschaftliche Arbeit von nicht-staatlichen Experten darauffolgend in politischen Prozessen von Repräsentanten der Staaten, die keine ethischen Experten sind, nichtig gemacht wurde. $^{37}$

Grundsätzlich wurden bei der Erarbeitung der Deklarationen immer Konsultationen geführt. Hierbei ist eine Entwicklung sowohl bei der Anzahl dieser als auch bei der Verschiedenheit eingebundener Individuen, Gruppen und Institutionen zu beobachten.

Bei den letzten beiden Deklarationen wurden nicht nur Experten unterschiedlicher Disziplinen, sondern auch Vertreter verschiedener Religionen und zunehmend nationale Ethikkommissionen eingebunden. Bei allen drei Deklarationen hatten bestimmte Internationale Organisationen und Nichtregierungsorganisationen die Möglichkeit, aufgrund von Einladungen teilzunehmen. Bei der Gruppe der eingebundenen Internationalen Organisationen sollen dies hauptsächlich Sonderorganisationen der UN gewesen sein, wie weitgehend kritisiert wurde. Bei den teilnehmenden Nichtregierungsorganisationen wurde jeweils die Vielfältigkeit bemängelt. Hinsichtlich ersterem bezweckte die UNESCO allerdings mit der Einladung der UN-Sonderorganisationen, die Kritik über ihr angebliches Monopolmandat für die Steuerung bioethischer Fragestellungen verstummen zu lassen. Im

\footnotetext{
${ }^{36}$ D. Benatar, Bioethics and Health and Human Rights: A Critical View, 32 Journal of Medical Ethics 1 (2006) $17 \mathrm{ff} ., 19 \mathrm{f}$.

${ }^{37}$ Für beide Meinungen siehe Langlois (Fn. 35), 56.
} 
Hinblick auf die bemängelte Vielfältigkeit der Nichtregierungsorganisationen wurde ihre mittlerweile fast nicht mehr zu überblickende Vielfältigkeit als Erklärung genannt. Trotz dieser Kritik gelang es der UNESCO, die wichtigsten Nichtregierungsorganisationen einzuladen und tatsächlich einzubinden. Hier ist vor allem bei der Einladung von Nichtregierungsorganisationen schutzbedürftiger Gruppen eine Verbesserung bei der Erarbeitung der dritten Deklaration zu beobachten.

Obwohl die Regierungsexperten in Form des IGBC und der abschließenden Expertenmeetings wesentliche Änderungen vorgenommen haben, wurden in diesen Phasen keine Konsultationen mehr geführt.

Die inhaltliche Entscheidungsfindung bei der Ausarbeitung der Deklarationen und ihren Inhalt selbst betreffend lässt sich Folgendes festhalten.

Die vielfältige und differenzierte Betrachtung der (genetischen) Zukunftsprobleme in den vorbereitenden Berichten des IBC konnte sich in den Erklärungen nicht vollständig durchsetzen. Der Einfluss der Regierungsexperten und -repräsentanten trug einerseits dazu bei, die verpflichtende Form der Deklarationen abzuschwächen.

In Bezug auf die Stellung der Person sind wenige konkrete Aussagen in den Deklarationen festgehalten worden, die genaueren Konturen der Stellung werden durch die den Betroffenen zugesprochenen Rechte erkennbar. In der ersten Deklaration wird die Stellung der Person mehr nach ihrer Leiblichkeit gewichtet und in der zweiten Deklaration mehr nach ihrer Normativität geprägt, die in der Vernunft und Freiheit wurzelt. In der dritten Deklaration wird die Stellung auch hauptsächlich an der Freiheit der Person orientiert beschrieben, wobei eine Ausformulierung des Integritätsschutzes auf universeller Ebene nicht gelungen ist - er blieb auf schutzbedürftige Gruppen beschränkt.

In den Deklarationen werden allgemeine Aussagen über die Rechte getroffen, welche unabhängig von den genetischen Eigenschaften respektiert werden sollen. Konkrete Rechte werden aber nur an sehr wenigen Stellen genannt, sie werden eher als ethische Prinzipien oder als Verbote an den Adressaten ausformuliert. Dies schwächt die Stellung der Person erheblich ab. Zudem fehlt vollständig die Kontextualisierung genetischer Anlageträgerschaften und Eigenschaften; die Reflexion des Arzt-Patienten-Verhältnisses, des familiären Kontextes und des breiteren Umfelds der Betroffenen (Arbeitgeber, Versicherungen) wird nicht geleistet. Neue biomedizinische Herausforderungen werden spezifisch - da dies abgelehnt wurde nicht wahrgenommen und es werden keine Hinweise für die Abwägung der Prinzipien für diese geleistet. Die während des Erarbeitungsprozesses geforderte Klärung bestimmter 
Grundbegriffe (wie der Begriff der Person) wurde mangels Einigkeit über diese nicht vorgenommen.

Die Bestimmung der Grundbegriffe und die Abwägung der Prinzipien werden zu oft im Arbeitsbereich nationaler Ethikkommissionen eingegliedert oder sollen aufgrund nationaler Regelungen und Vorschriften erfolgen. Somit werden die notwendige Internationalität und damit eine umfassende und auf universeller Ebene adäquat konturierte Stellung der Person nicht geleistet.

Die Bindungskraft der Instrumente ist wie folgt zu bewerten.

Die drei Deklarationen der UNESCO sind als Soft Law einzustufen. Sie stellen internationale und konsensuale Mindeststandards in der Biomedizin und der Bioethik dar und verkörpern einen Aufruf an die internationale Gemeinschaft, die ethischen Dilemmas in Bezug auf das menschliche Genom, genetische Daten und die Bioethik im Allgemeinen im Rahmen der verrechtlichten Menschenrechte zu berücksichtigen. ${ }^{38}$

Die Frage, ob die Menschenrechte durch die Einbindung ethischer Prinzipien in ihren Referenzrahmen ein ethisches Minimum darstellen, wurde von der UNESCO oder von ihren Komitees nicht unmittelbar angesprochen. Die abschließenden Artikel der jeweiligen Deklarationen untersagen eine Auslegung der Prinzipien, die den Menschenrechten und Grundfreiheiten zuwiderläuft. Demnach können die Prinzipien nur im universellen menschenrechtlichen Rahmen interpretiert werden. Somit kann ein ethischer Pluralismus nur insoweit durchgesetzt werden, als dieser mit den Menschenrechten im Einklang steht. Dies spricht zunächst für eine Betrachtung des menschenrechtlichen Referenzrahmens als ein bioethisches Minimum. ${ }^{39}$

Bei der Förderung und Umsetzung der Deklaration gewinnen die Staaten eine immer größer werdende Rolle, nicht nur bei der Organisation ethischer Bildung, sondern durch die Verpflichtung, internationale Kooperationen insbesondere mit Entwicklungsländern einzugehen. Die Aufgaben und der Einfluss des IBC werden weniger; zuerst wird im Vergleich zur ersten Deklaration der IGBC als gleicher Partner bei der Förderung und Umsetzung genannt, danach werden in der dritten Deklaration beide Komitees lediglich als Hilfsorganisationen der UNESCO ohne konkrete Aufgaben erwähnt. Die nationalen

\footnotetext{
${ }^{38}$ E. Benda, Interview in Frankfurter Rundschau, 1. November 1997, 1.

${ }^{39}$ Weitere Argumente für und gegen eine Befassung mit Bioethik in einem rechtlichen Sprachreferenzrahmen siehe M. Clados, Chances for and Limits of International Law and Legal Language in the Area of Bioethics, in: S. Vöneky/C. Hagedorn/M. Clados et al. (Hrsg.), Legitimation ethischer Entscheidungen im Recht. Interdisziplinäre Untersuchungen, Springer, Berlin/Heidelberg 2009, 297 ff., 312 ff.
} 
Ethikkommissionen bekommen dagegen eine bedeutende Rolle bei der Umsetzung der Deklarationen, vor allem aber im Rahmen der staatlichen Förderung der Umsetzung. Diese Tendenzen, die Verlagerung der Zuständigkeiten der UNESCO-Komitees auf die nationalen Ethikkommissionen einerseits und die zunehmende Verpflichtung der Staaten andererseits, scheinen aber doch gegen das deklarierte Ziel der UNESCO zu laufen, überwölbende ethische Prinzipien unter den Konzepten der Menschenwürde und Menschenrechte auf globaler Ebene zu verabschieden und die Rolle eines internationalen Koordinators bei ihrer Förderung und Umsetzung zu übernehmen. ${ }^{40}$ Die zunehmende Inpflichtnahme der Staaten bleibt aufgrund der schwachen Formulierung (,should“) und durch den an vielen Stellen eingeräumten Vorrang nationaler Vorschriften, zudem ohne die Verabschiedung jeglicher Berichterstattungspflicht oder eines Kontrollmechanismus, relativ wirkungslos.

Im Ergebnis lässt sich festhalten: Weil die bisherigen Handlungs- und Regelungsempfehlungen der UNESCO die Stellung der Person für den Kontext einer vollstandigen Sequenzierung des Genoms spezifisch nicht weiterentwickeln sind sie nicht geeignet, die Stellung des Patienten in diesem neuen Kontext angemessen zu reflektieren. Um dies bei einem potenziell neuen Instrument der UNESCO doch zu erreichen, werden im kommenden Teil der Arbeit Änderungsvorschläge vorgetragen. Vor allem wird der Frage nachgegangen, wie man die Faktoren, die einen Einfluss auf die Legitimität der Steuerung haben, so gestaltet, dass diese die Herausforderungen und Eigenschaften der Ganzgenomanalyse angemessen berücksichtigen und somit insgesamt eine Anerkennungswürdigkeit der Steuerung herbeiführen (Kapitel 8: Genetische Analysen im internationalen Menschenrechtssystem auf universeller Ebene - Bewertung de lege lata, auf dem Weg zu de lege ferenda).

Die Anerkennungswürdigkeit einer Deklaration kann zunächst kraft Beteiligung an der Entscheidungsfindung sowie kraft eines effizienten Entscheidungsfindungsverfahrens gesteigert werden (I. Verbesserung der deliberativen Prozesse: Repräsentation,

\section{Entscheidungsfindung).}

Zu der Beteiligung an den Arbeitsgruppen im Rahmen des IBC-, IGBC- und der IGE-Treffen lässt sich in Hinblick auf eine künftige Deklaration Folgendes festhalten: Der Auftrag des IBC, die ethische Auseinandersetzung mit den lebenswissenschaftlichen Entwicklungen zu

\footnotetext{
${ }^{40}$ UNESCO IBC, Human Genetic Data: Preliminary Studie by the IBC on its Collection, Processing, Storage and Use, SHS-503/01/CIB-8/3 (Rev.2), Paris, 15.05.2002, 1, I.6.
} 
gewährleisten, soll weiterhin im Fokus bleiben. Mithin wäre in der Zukunft darauf zu achten, bei der Zusammenstellung des Komitees Wissenschaftler einzuberufen, die aufgrund einer ausgezeichneten Lebensleistung als etabliert gelten. Ein Vorteil wäre die Vermeidung einer Einberufung von Mitgliedern mit politisch-diplomatischem Hintergrund in diese Ethikkommission. Da die Mitglieder in ihren persönlichen Funktionen arbeiten sollten, wäre es überlegenswert, ihre Nominierung nicht den Mitgliedstaaten zu überlassen. Um die Arbeit $\mathrm{zu}$ intensivieren und eine zeitnahe Reflexion der Lebenswissenschaften zu ermöglichen, könnten Tagungen des Komitees mehrmals im Jahr vorgeschrieben werden. Für die Gewährleistung des ethischen Pluralismus ist die Beibehaltung der Möglichkeit wichtig, abweichende Meinungen in den sonst durch Konsens zu verabschiedenden Berichten und Empfehlungen veröffentlichen zu können.

Die Hauptrolle des IGBC, eine zeitnahe politische Kontrolle der Arbeit des IBC zu gewährleisten, kann angesichts seiner Zusammensetzung nicht ganz nachvollzogen werden. Unabhängig von der Bewertung seiner Arbeit selbst kann die Einbindung des IGBC als Repräsentant und Filter der Mitgliedstaaten an erster Stelle auch aus diesem Grund oft nur eine willkürliche politische Urteilsfindung über die Arbeit des IBC leisten. In dem Komitee sind jeweils nur ein Fünftel aller Mitgliedstaaten repräsentiert. Zudem entsprechen die vorhandenen Sitze nicht der Zahl der Mitgliedstaaten in der jeweiligen geographischen Region. $^{41}$ Folglich können die Entscheidungen keine geographisch ausgeglichenen Mehrheitsentscheidungen darstellen.

Bei den beiden abschließenden IGE-Treffen wird die geographische Ausgeglichenheit theoretisch gewährleistet, denn alle Staaten können zu diesen Treffen Repräsentanten schicken. Die Teilnahme war in den letzten Jahren allerdings nicht ausgeglichen. ${ }^{42}$ Die größeren Gruppen stammten immer aus den Industrieländern. ${ }^{43}$ Während dieser Treffen wird

\footnotetext{
${ }^{41}$ Die Sitzverteilung und die Wahl sollen eigentlich entsprechend der Entscheidung des Exekutivrates erfolgen: 155 EX/Decision 9.2, Paris, 03.12.1998. Demnach hat die Gruppe I (Westeuropa und die Nordamerikanischen Staaten) sieben, die Gruppe II (Osteuropa) vier, die Gruppe III (Lateinamerika und Karibikstaaten) sechs, die Gruppe IV (Asien und die Pazifikstaaten) sieben und die Gruppe V (Afrika (acht) und die arabischen Staaten (vier)) insgesamt zwölf Sitzplätze. Für die regionale Gruppierung der UNESCO siehe http://www.unesco.org/new/en/unesco/worldwide/ (aufgerufen am 30.11.2014).

${ }^{42}$ R. Andorno, Seeking Common Ground on Genetic Issues: The UNESCO Declaration on the Human Genome, in: J. Sándor (Hrsg.), Society and Genetic Information. Codes and Laws in the Genetic Era, Central European University Press, Budapest/New York 2003, 105 ff., 106. UNESCO, Meeting of Government Experts Responsible for Finalizing the Draft International Declaration on Human Genetic Data: final report, Paris, 28.05.2003, SHS/EST/03/CONF.203/6, 1; Langlois (Fn. 35), 48.

43 UNESCO, First Intergovernmental Meeting of Experts Aimed at Finalizing a Draft of a Declaration on Universal Norms on Bioethics: list of participants, 06.04.2005, Paris, SHS/EST/05/CONF.203./INF.1; Second
} 
jeweils eine gerechte Verteilung der Stimmen angestrebt. ${ }^{44}$ Bei der vorhandenen Beteiligung der Ländergruppen an den Treffen und bei der unterschiedlichen Mitgliederzahl der Delegiertengruppen relativiert sich aber die Bedeutung der Verfahrensordnung.

$\mathrm{Zu}$ der Organisation und zu dem Verfahren der Konsultationen mit Akteuren außerhalb und innerhalb der UN lässt sich Folgendes feststellen:

Entsteht der Anspruch auf die internationale Steuerung genetischer Analysen, so sollen zuerst Bemühungen angestellt werden, das hierfür notwendige Verfügungswissen einzuholen. Wenn eine genaue Analyse der Handlungsketten der Arbeitsprozesse vorliegt und die Eigenschaften einer neuen Technologie in Bezug auf ihre Herausforderungen ausführlich erfasst wurden, kann diese Grundlage den Diskursprozessen Glaubwürdigkeit verleihen. Der IBC kann als erste Stelle für die Erfassung des Verfügungswissens funktionieren, weil dies seiner Aufgabenzuteilung entspricht.

Um dem Anspruch der Aktualität bei den gegenwärtigen Arbeiten und Fokussierungen auf die Genetik gerecht zu werden, wäre eine effektivere Einbindung der internationalen Akteure der translationalen Medizin in den Entscheidungsfindungsprozess angebracht. Obwohl dem IBC ständig zwei Mitglieder angehörten, die auch in der Human Genome Organization engagiert waren, sollten künftig auch die weiteren Interessengruppen in der translationalen Genomforschung repräsentiert werden. Angesichts der Legitimation der Entscheidungen ist dies vor allem wichtig, da diese Akteure später Adressaten eines verabschiedeten Instruments werden können. ${ }^{45}$

Die Konsultation mit Organisationen innerhalb des UN-Systems kann ebenfalls verbessert werden. Obwohl die verstärkte Einbindung weiterer UN-Sonderorganisationen oft die Kritik erntete, Entscheidungsfindungen über biomedizinische Fragen stünden ausschließlich der UNESCO $\mathrm{zu}$, ist dies nicht berechtigt. ${ }^{46}$ Eine flächendeckende Beschäftigung mit biomedizinischen Fragen innerhalb des UN-Systems kann die Einbindung der Lebenswissenschaften in den menschenrechtlichen Referenzrahmen unterstreichen. Sie steigert auch die Glaubwürdigkeit, wenn innerhalb des UN-Systems eine breite

Session of the Intergovernmental Meeting of Experts Aimed at Finalizing a Draft of a Declaration on Universal Norms on Bioethics: list of participants, Paris, 25.06.2005, SHS/EST/05/CONF.204./INF.1.

${ }^{44}$ UNESCO, First Intergovernmental Meeting of Experts Aimed at Finalizing a Draft Declaration on Universal Norms on Bioethics: provisional rules of procedure, Paris, 04.02.2005, SHS/EST/05/CONF.203/2 (Prov.), 3; Second Session of the Intergovernmental Meeting of Experts Aimed at Finalizing a Draft Declaration on Universal Norms on Bioethics: rules of procedure, Paris, 04.05.2005, SHS/EST/05/CONF.204/2, 3.

45 J. Friedrich, International Environmental ,soft law“, Springer/Heidelberg u.a. 2013, 373 ff., 389.

${ }^{46}$ R. Ashcroft, The troubled Relationship Between Bioethics and Human Rights, in: M. Freeman, Law and Bioethics. Current Legal Issues, Vol. 11, OUP, Oxford 2008, 31 ff., 37. 
Auseinandersetzung mit dem Thema stattfindet. Um wiederum die Doppelung der Arbeit und einen Wettbewerb zwischen den Organisationen für die Steuerung der Biomedizin zu unterbinden, sollten ihre einzelnen Zuständigkeiten klar festgelegt und voneinander abgegrenzt werden.

Das internationale Soft Law ist in erster Linie dazu geeignet, die Normierung der Biomedizin und der Lebenswissenschaften auf globaler Ebene zu begleiten. Obwohl die Schwierigkeiten der Verabschiedung eines verbindlichen Instruments während der Erarbeitungsprozesse erkannt wurden, wurde die Verbindlichkeit an sich als wünschenswert empfunden (II. Die Normativität und die Bindungskraft der Instrumente: 1. Bestrebung nach bindender Kraft und der Einfluss deklaratorischer Instrumente auf das Völkerrecht).

Bei der Bestrebung nach völkerrechtlicher Verbindlichkeit soll vor allem auf die Eigenschaften des Soft Law abgestellt werden, Einfluss auf das Völkerrecht ausüben und dadurch mittelbar zur Erzeugung verbindlicher Vorschriften beitragen zu können. Als Beispiel hierfür ist der Kodex der FAO, der Code of Conduct for Responsible Fisheries [CCRF] zu nennen. ${ }^{47}$

Will man außerdem an den Vorteilen von Soft Law - an der schnellen Verabschiedung, an der Bereitschaft der Mitgliedstaaten, sich über nicht verbindliche Normen zu einigen, an der Flexibilität des Instruments, aber vor allem an der zeitnahen faktischen Bindungswirkung der Deklarationen - festhalten, so gibt es verschiedene Wege, die bereits verabschiedeten Deklarationen selbst in ihrer außerrechtlichen Bindungswirkung weiterzuentwickeln oder eine neue Deklaration mit stärkerer außerrechtlicher Bindungswirkung aufzurüsten (2. Stärkung der außerrechtlichen Bindungswirkung der Instrumente). Einerseits kann die faktische Bindungswirkung durch die angemessene Einbindung der Ethik als eine Disziplin, die an erster Stelle das Handeln außerhalb des Bereichs des Rechts, also überpositiv leitet und rechtfertigt, erhöht werden. Andererseits kann sie möglicherweise durch die Einbindung von bereits existierenden internationalen Maßstäben für die Biomedizin gesteigert.

Zwei Wege scheinen für die Einbindung der die Praxis leitenden Instrumente sinnvoll. Öffnungsklauseln stellen die erste Möglichkeit für die Einbindung existierender Kodizes und Richtlinien dar. Der Einsatz von Öffnungsklauseln ist nur unter der Erfüllung von zwei

${ }^{47}$ Food and Agriculture Organization (FAO), Code of Conduct for Responsible Fisheries (CCRF), Rom 1995. Siehe den Text des Kodexes: Report of the Conference of FAO, Twenty-Eighth Session, 20.-31. Oktober 1995, Annex 1 oder unter http://www.fao.org/docrep/005/v9878e/v9878e00.htm (aufgerufen am 30.11.2014). 
Kriterien angemessen. Erstens müssen die Prinzipien der Kodizes und Richtlinien ethisch gerechtfertigt werden und von Standards der Berufsmoral klar abgegrenzt werden. Zweitens dürfen die so einbezogenen Standards das Völkerrecht nicht verletzen. ${ }^{48}$

Diese Klauseln werden in der Regel aber allgemein ausformuliert und räumen grundsätzlich keine Möglichkeit ein, zwischen den einzubindenden Instrumenten zu unterscheiden. Eine pauschale Annahme verschiedenster Leitlinien und Richtlinien ohne vorherige sorgfältige Prüfung kann nicht wünschenswert sein.

Der zweite Weg könnte über die jeweilige Präambel führen, indem diese auf wichtige und von der UNESCO geprüfte Kodizes verweisen würde. Präambel tragen zur Bestimmung des Zwecks des Instruments bei, weil sie dabei helfen, die Interpretation des Textes zu gewährleisten und seine Bedeutung zu erfassen. ${ }^{49}$ Die Erfassung der Bedeutung des Instruments und die Bestimmung seines Zwecks könnten auch die Einbindung der existierenden Kodizes und Richtlinien, die auf internationaler Ebene die translationale medizinische Praxis bestimmen, umfassen.

Durch die Einbindung einschlägiger internationaler Kodizes und Leitlinien würden die SoftLaw-Dokumente an Bestimmtheit gewinnen und könnten dadurch ihre Rolle als Interpretationshilfe für das Völkerrecht und für die nationalen Rechtsvorschriften ${ }^{50}$ auch besser erfüllen.

Die Einbindung der Ethik gelingt in den UNESCO-Deklarationen vorwiegend durch die unmittelbare Annahme von ethischen Prinzipien.

Das für die Umsetzung des ethischen Mandats zuständige Organ der UNESCO ist der IBC. Durch die Zusammenführung der Arbeit des IBC mit der des IGBC und durch die Finalisierung der Deklarationen durch die IGE-Treffen demonstriert die UNESCO die Entwicklung der Bioethik aus einer akademischen Disziplin heraus hin zu einem Feld der Politik. ${ }^{51}$ Die Deklarationen bewegen sich durch ihre Erarbeitungskonstellation in einer Grauzone der Politik, sie sind an erster Stelle Elemente und Ergebnisse politischer

\footnotetext{
${ }^{48} \mathrm{Zu}$ diesen Kriterien, aber auch zur Analyse von Öffnungsklauseln im deutschen Recht siehe S. Vöneky, Ethische Standards im Wissenschaftsrecht, in: W. Löwer/K. F. Gärditz (Hrsg.), Wissenschaft und Ethik, Wissenschaftsrecht: Zeitschrift für deutsches und europäisches Wissenschaftsrecht, Beiheft 21/2012, $68 \mathrm{ff}$.

${ }^{49}$ H. Gros Espiell, The Preamble, in: H. ten Have/M. Stanton Jean (Hrsg.), The UNESCO Universal Declaration on Bioethics and Human Rights. Background, principles and application, UNESCO, Paris 2009, 17 ff., 57 ff., 58 f.

${ }^{50}$ D. Thürer, Soft law, in: R. Wolfrum (Hrsg.), MPEPIL, März 2009, Rn. 29 f.

${ }^{51}$ H. ten Have, Kulturübergreifende Bioethik. Der Ansatz der UNESCO, in: L. Honnefelder/D. Lanzerath (Hrsg.), Bioethik im Kontext von Recht, Moral und Kultur. Beiträge der Internationalen Konferenz vom 14.15.12.2005 in Berlin, University Press, Bonn 2008, 123 ff., 125.
} 
Entscheidungen. ${ }^{52}$ Kritisch kann aus diesem Grund das Wesen der Deklarationen als Sammlung universeller ethischer Prinzipien in Frage gestellt werden, denn die Dokumente werden dem Anspruch nicht gerecht, universelle ethische Standards zu verabschieden.

Einerseits würde die Vorlagerung der ethischen Entscheidungsfindung vor der politischen den Ursprüngen der Bioethik gerecht, die in der Moralphilosophie und nicht in der politischen Philosophie $\mathrm{zu}$ finden sind. $^{53}$ Andererseits würde die Trennung der ethischen Entscheidungsfindung von den politischen Abwägungen eine noch bessere Fokussierung auf die traditionellen Problembereiche der medizinischen Ethik erlauben, die aus der Betrachtung des Arzt-Patienten-Verhältnisses und der neuen technologischen Herausforderungen resultieren. $^{54}$

Das Menschenrechtssystem ist das einzige bewährte System, das zur Verfügung steht, um den Menschen auf globaler Ebene verbindlich zu schützen. Der juristische Referenzrahmen der Menschenrechte erlaubt die grundsätzliche Strukturierung spezifischer normativer Fragen. ${ }^{55}$ Dies rechtfertigt, bestimmte Prinzipien in ihren Referenzrahmen zu integrieren. ${ }^{56}$ Dem von der UNESCO verfolgten Ansatz, die Bioethik im rechtlichen Referenzrahmen der Menschenwürde und Menschenrechte zu handhaben, ist daher grundsätzlich zuzustimmen.

Eine Einbindung in den menschenrechtlichen Referenzrahmen könnte neben Hinweisen auf Völkerrechtsverträge, wie dies bei den bereits bestehenden UNESCO-Deklarationen der Fall ist, auch durch zusätzliche Hinweise auf ihre Vertragsorgane geschehen. Diese Organe können selbstverständlich auch dann aktiv werden, wenn die durch den bestimmten Vertrag geschützten Menschenrechte in Verbindung mit der Biomedizin oder einer biotechnologischen Anwendung verletzt werden. ${ }^{57}$

\footnotetext{
${ }^{52}$ Siehe Andorno (Fn. 42), 106.

${ }^{53}$ D. Mieth/M. Düwell, Ethische Überlegungen zur UNESCO-Deklaration über das menschliche Genom und die Menschenrechte, in: D. Mieth (Hrsg.), Was wollen wir können? Ethik im Zeitalter der Biotechnik, Herder, Freiburg/Basel/Wien 2002, 344 ff., 347.

${ }^{54}$ Siehe allerdings den Verweis auf Dissense über ethische Prinzipien in einer pluralistischen Weltgesellschaft als politische Aufgabe bei S. Vöneky, Grundlagen und Grenzen der Ethisierung des Völkerrechts, in: Dies./B. Beylage-Haarmann/A. Höfelmeir et al., Ethik und Recht - Die Ethisierung des Rechts, Springer, Heidelberg u.a. 2013, 129 ff., 142.

${ }^{55}$ P. Farmer/N. G. Campos, New Malaise: Bioethics and Human Rights in the Global Era, 32 Journal of Law, Medicine and Ethics 2 (2004) 243 ff., 248.

${ }^{56} R$. Andorno, Biomedicine and international human rights law: in search of a global consensus, 80 Bulletin of the World Health Organization 12 (2002) 959 ff., 960.

${ }^{57}$ Eine „Konturierung“ im Bereich der Menschenrechte aufgrund der Arbeit der Vertragsorgane bestätigt $E$. Klein, Impact of Treaty Bodies on the International Legal Order, in: R. Wolfrum/V. Röben (Hrsg.), Developments of International Law in Treaty Making, Springer, Berlin/Heidelberg 2005, 571 ff., 578.
} 
Bei der Einbindung in den menschenrechtlichen Referenzrahmen soll das Biorecht die Bioethik allerdings nicht subsumieren. Dies kann tatsächlich nur dann erfolgen, wenn die Räume der ethischen Bewertung zunächst von der politischen klar abgegrenzt werden. Folglich sollte die Arbeit des IBC von der Politik möglichst abgegrenzt werden. Wenn der Anspruch lautet, universelle ethische Prinzipien zu formulieren, kann die Etablierung von Ethikkommissionen auf mitgliedstaatlicher Ebene diese Trennung nicht ersetzen. Die ethische Argumentation des IBC und die Eingliederung der von ihm verabschiedeten Prinzipien in den menschenrechtlichen Referezrahmen gehen sonst verloren.

Überlegenswert wäre es, die etablierten Organe des UN-Menschenrechtsschutzsystems in die rechtliche Konkretisierung der Menschenrechte vor Verabschiedung einer künftigen Deklaration einzubinden. Angesichts des Auftrags des UN-Menschenrechtsrates [UNHRC] und angesichts der nicht minderen Kritik am UN-Menschenrechtsschutzsystem, nach welcher $\mathrm{zu}$ viele Organe mit überlappenden Aufgabenbereichen in diesem tätig sind ${ }^{58}$, würde ein besonderer Auftrag für den Rat in Bezug auf die Konkretisierung der Patientenrechte im Erarbeitungsprozess biomedizinischer Instrumente der UNESCO eine Bereicherung für diese Dokumente darstellen. Inwieweit dann die Arbeit des IGBC noch als notwendig anzusehen wäre, sollte zusätzlicher Prüfung unterzogen werden. Die Zustimmung zweier finalisierender Treffen der Regierungsexperten würde ausreichen, um die politischen Interessen der Mitgliedstaaten nach der ethischen und rechtlichen Arbeit in den Instrumenten abzubilden.

Bei der Eruierung über die Möglichkeiten der Steigerung der thematischen Effektivität eines künftigen Instruments zur genetischen Analyse wird die Person in den Mittelpunkt gestellt (IV. Steigerung der thematischen Effektivität).

Die Beschreibung der Person aufgrund von sie kennzeichnenden Merkmalen als Möglichkeiten, welche im Laufe der Entwicklung der drei Deklarationen immer weiter ausformuliert wurde, kann ein erfolgreicher Ansatz werden, um trotz abweichender moralischer Auffassungen und verschiedener legislativer Lösungen in den Mitgliedstaaten eine gemeinsame Vorstellung über die Stellung des Patienten zu entwickeln.

Die Kritik, nach der eine Definition der Person fehlt, ist nicht berechtigt. Um moralische und juristische Stellungen auf universeller Ebene $\mathrm{zu}$ begründen, ist die Identifizierung von Aspekten des Personenseins, die Bestandteile verschiedener Konzepte sein können, erforderlich, nicht aber ein vollständiges und abgeschlossenes Konzept des Personenseins

\footnotetext{
${ }^{58}$ M. G. Schmidt, Reform of the United Nations Human Rights Programs - current challenges and trends, in: $H$. Volger/N. Weiß (Hrsg.), Die Vereinten Nationen vor globalen Herausforderungen, Referate der Potsdamer UNOKonferenzen 2000-2008, Universitätsverlag, Potsdam 2011, 81 ff., 82 f.
} 
selbst. ${ }^{59}$ Die Frage, welche Stellung dem Patienten zukommen soll, kann auf überstaatlicher Ebene geklärt werden. Wie diese Stellung zu verwirklichen ist, bleibt eine Aufgabe der Mitgliedstaaten, die dabei ihre rechtsystematischen und kulturspezifischen Besonderheiten zu berücksichtigen wissen werden. ${ }^{60}$

Vor allem soll in einer künftigen Deklaration, welche Antworten auf die Herausforderungen neuer genetischer Analysemethoden anbietet, ein Zusammenhang zwischen den besonderen Eigenschaften genetischer Informationen und der persönlichen Identität und Integrität hergestellt werden. Die Genetifizierungs- und Medikalisierungsgefahr aufgrund prädiktiver genetischer Informationen betrifft jeden. Demnach ist die Eingrenzung des Integritätsschutzes auf besondere schutzbedürftige Gruppen nicht zu rechtfertigen. Die Beibehaltung und - im Vergleich zu der zweiten Deklaration - die Erweiterung eines „Vokabulars“ biologischer Begriffe (u.a. mit der Definition des Epigenoms) kann für die Klärung des Zusammenhanges zwischen dem Genom und der personalen Integrität und Identität ebenfalls förderlich sein, da aufgrund der Erläuterung genetischer Funktionen ein genetischer Determinismus erfolgreich und glaubwürdiger abgelehnt werden kann.

Um die spezifischen Eigenschaften der Genomanalyse zu berücksichtigen, sollte eine künftige Deklaration Lösungen für ihre besonderen Herausforderungen finden. Neben der Identität und Integrität sind weitere Aspekte für die Stellung des Patienten als Person im genetischen Kontext tragend, die im Vergleich zu den bisher verabschiedeten Aspekten zusätzlich erwähnt, besser hervorgehoben oder ausführlicher konkretisiert werden sollten. Zu diesen Aspekten gehören die genetische Identifizierbarkeit, die Unverfügbarkeit der menschlichen Erbinformation, die Speicherung genetischer Daten, das Recht, die eigene Erbinformation an die Nachkommen weitergeben zu dürfen, der Schutz genetischer Diversität, ein eindeutiges Patentierungsverbot und Kommerzialisierungsverbot oder ein Verbot der Keimbahnmanipulation zu nicht-therapeutischen Zwecken.

Um die Stellung der Patienten im Kontext genetischer Analysen zu konkretisieren und sie in einem künftigen Dokument durch eine klare rechtliche Stellung zu schützen, sollten die Aspekte der Personenstellung, die Schutzbereiche der verrechtlichten Menschenwürde und

\footnotetext{
${ }^{59}$ J. D. Ohlin, Is the concept of the person necessary for human rights? 105 Columbia Law Review 1 (2005) 209 ff.

${ }^{60}$ Für eine Betrachtung der internationalen Harmonisation auf moralisch problematischen Gebieten siehe $M$. Friele, Striving for Harmonisation and Living Without it - Is International Legislative Harmonisation in Morally Problematic Areas such as Research on Human Embryos Ethically Justifiable? In: S. Vöneky/C. Hagedorn/M. Clados et al. (Fn. 39), $331 \mathrm{ff}$.
} 
der Menschenrechte betreffen, nicht als Prinzipien ausformuliert, sondern eindeutig als Rechte identifiziert und ausformuliert werden. Die letzte Konkretisierung findet zwar im politischen Verhandlungsprozess der IGE-Treffen statt, es darf dennoch nicht untergehen, dass das $\mathrm{zu}$ verabschiedende Instrument den Adressaten Vorschläge für die juristische Auslegung der Menschenrechte in einem spezifischen Kontext zu vermitteln hat und sich weder mit gemeinsamen ethisch-moralischen Überzeugungen benügt noch mit politischen Interessen erheblich aufgeweicht wird. Dies schließt eine Aufnahme moralischer Prinzipien, die den Rechten vorausgehen, oder die Ausformulierung von Prinzipien, die moralische Gebote mit den Menschenrechtsnormen verbinden, nicht aus.

Wie dann die konkretisierten Patientenrechte selbst einzustufen sind - als eine Spezifizierung der Menschenrechte, eine neue Generation dieser oder sogar als internationale subjektive Rechte - wird von der Entwicklung der Biomedizin selbst bestimmt werden. Der zunehmend interindividuelle Charakter biomedizinischer Patientenrechte und die Einbindung der Medizinethik in ihre Bestimmung sprechen zwar auf den ersten Blick für die Entwicklung eines dynamischen Bestands an Rechten; die Rolle dieser Rechte beim Schutz der Würde und der personellen Identität und Freiheit des Patienten unterstreicht aber zunehmend ihren Menschenrechtscharakter.

Bei der Betrachtung der Förderung und Umsetzung eines künftigen Instruments ist zunächst zu unterscheiden, ob diese leichter anhand von durch Adressaten durchgeführter Maßnahmen oder besser anhand von Durchsetzungsmechanismen der UNESCO selbst ausgeführt werden sollen (V. Bessere Förderung und Umsetzung).

Um die Durchsetzung zu fördern, können zunächst im Dokument selbst Verpflichtungen für die Adressaten verabschiedet werden. Bei einer unverbindlichen Deklaration hängt die Erfüllung der Berichterstattungspflicht vom Willen der Staaten ab. Da nicht-verbindliche Instrumente einen großen Spielraum für die Auslegung ihrer Vorschriften einräumen, darf die Berichterstattung über die tatsächliche Umsetzung theoretisch keine großen Mühen für die Staaten bedeuten. ${ }^{61}$ Wenn die Bereitschaft fehlt, über die Förderung und Umsetzung der Vorschriften zu berichten, könnten Überwachungsmechanismen erfolgreiche Unterstützung anbieten. Die Frage allerdings, wer die Aufsichtsrolle erfüllen könnte, ist nicht einfach zu beantworten, weil im Rahmen der bisherigen UNESCO-Deklarationen oder in nachfolgenden Resolutionen solche Mechanismen nicht verabschiedet wurden. Zurzeit verfügt die UNESCO

\footnotetext{
${ }^{61}$ O. S. Stokke, Regimes as Governance Systems, in: O. R. Young (Hrsg.), Global Governance: drawing insights from the environmental experience, MIT Press, Cambridge, MA u.a. 1997, 27 ff., 50.
} 
über eine der schwächsten Überwachungsmechanismen unter den Internationalen Organisationen. ${ }^{62}$ Bei der Einführung eines Überwachungsmechanismus könnten sowohl die Maßnahmen für die Überwachung der Umsetzung des CCRF als auch Überwachungsmechanismen im internationalen Wirtschaftsrecht, die Leitlinien der Organisation für Wirtscahftliche Zusammenarbeit und Entwicklung [OECD] für Multinationale Unternehmen als Beispiel, fungieren. ${ }^{63}$ Demnach könnte die Berichterstattung der Adressaten zumindest mit einem Auditierungsprozess kombiniert werden, mithilfe dessen die Berichte durch ein unabhängiges Organ überprüft werden. ${ }^{64}$ Im Rahmen der Auditierung könnte das Aufsichtsorgan zudem eine Veröffentlichung seiner Ergebnisse anstreben.

Auch das Global Ethics Observatory [GEObs], ein System von Datenbanken im Bereich der angewandten Ethik, könnte zu einem Kontrollmechanismus weiterentwickelt werden, wenn die Mitgliedstaaten verpflichtet wären, ihre legislativen Maßnahmen in dieser Datenbank zu speichern und mit den Vorschriften eines künftigen Instruments zu verbinden. ${ }^{65}$ Indem diese Verlinkung nicht wie gegenwärtig vom Sekretariat der UNESCO erstellt würde, könnte sie als eine Art Berichterstattung funktionieren und den Prozess, technische und wissenschaftliche Fortschritte mit Regelungen auf nationalen Ebenen zu begleiten, beschleunigen. ${ }^{66}$

Sollten die Adressaten unwillentlich nicht in der Lage sein, den Vorschriften zu folgen, dann wäre ein strukturiert geleitetes Management für die Umsetzung angemessen. In diesem Bereich leistet die UNESCO im Rahmen ihrer globalen Ethikprogramme bereits sehr viel. GEObs ist eine große Hoffnung auch bei dem Management der Umsetzung, denn sie bietet auch eine Verlinkung der rechtlichen Dokumente mit den Artikeln der Deklarationen 2003 und 2005 an, um als Beispiel oder Muster für Staaten zu dienen, die noch vor einer Umsetzung der Deklarationen in ihren nationalen Rechtssystemen stehen. ${ }^{67} \mathrm{Um}$ dabei wirklich hilfreich zu sein, sollten in der Datenbank auch die erste und eine womöglich künftige Deklaration sowie Referenzen zwischen den beiden ergänzt werden.

\footnotetext{
${ }^{62}$ R. Bank/F. Foltz, United Nations Educational, Scientific and Cultural Organization (UNESCO), in: Wolfrum (Hrsg.), MPEPIL, August 2010, Rn. 70 f.

${ }^{63}$ FAO, CCRF (Fn. 50); OECD, Guidelines for Multinational Enterprises, (1976) 15 ILM 969, die letzte aktualisierte Fassung ist abrufbar unter http://www.oecd.org/corporate/mne/48004323.pdf (aufgerufen am 30.11.2014).

${ }^{64}$ A. L. Taylor, Globalization and biotechnology: UNESCO and an International Strategy to Advance Human Rights and Public Health, 25 American Journal of Law and Medicine 4 (1999) 479 ff., 480, 513, 527,535 f.

${ }_{65} \quad$ Siehe http://www.unesco.org/new/en/social-and-human-sciences/themes/global-ethics-observatory/ (aufgerufen am 18.05.2015).

${ }^{66}$ Langlois (Fn. 35), 77, 90.

${ }^{67}$ T. W. Ang/H. ten Have/J. H. Solbakk et al., Unesco Global Ethics Observatory: Database on Ethics related legislation and guidelines, 34 Journal of Medical Ethics 10 (2008) 738 ff., 740.
} 


\section{Zusammenfassende Schlussbetrachtung}

In der vorgelegten Arbeit werden die Eigenschaften und die mit diesen einhergehenden normativen Herausforderungen der Gesamtgenomanalyse in der translationalen Medizin dargestellt. Als Grundlage und Vorsatz hat sich die Maxime der Stellung des Patienten als Person bestätigt und es konnte aufgezeigt werden, dass ihre Aufrechterhaltung und Konkretisierung im Kontext neuer genetischer Analysen zahlreiche Faktoren der Anerkennungswürdigkeit einer internationalen Steuerung beeinflusst. Demnach sind die Entscheidungsfindung, die Verbindlichkeit, die inhaltliche Effektivität sowie die Umsetzung der Empfehlungen und Vorschriften betroffen.

$\mathrm{Ob}$ die Grundlage für und der Zweck der Steigerung der Anerkennungswürdigkeit internationaler Regelungen der Biomedizin, die Aufrechterhaltung der Maxime einer Stellung des Patienten als Person bei der Steuerung biomedizinischer Fragen künftig gelingt, wird Maß und Grenze neuer Wege des Umgangs mit den Herausforderungen auf internationaler Ebene sein. 


\section{Literaturverzeichnis}

Andorno, Roberto Biomedicine and international human rights law: in search of a global consensus, 80 Bulletin of the World Health Organization 12 (2002) $959 \mathrm{ff}$.

Andorno, Roberto Seeking Common Ground on Genetic Issues: The UNESCO Declaration on the Human Genome, in: Judit Sándor (Hrsg.), Society and Genetic Information. Codes and Laws in the Genetic Era, Central European University Press, Budapest/New York 2003, 105 ff.

Ang, Tee Wee/ten Unesco Global Ethics Observatory: Database on Ethics related Have, Henk/ legislation and guidelines, 34 Journal of Medical Ethics 10 (2008) Solbakk, Jan Helgel $738 \mathrm{ff}$.

Nys, Herman

Ashcroft, Richard The troubled Relationship Between Bioethics and Human Rights, in: Michael Freeman, Law and Bioethics. Current Legal Issues, Vol. 11, OUP, Oxford 2008.

Bank, Roland/Foltz, United Nations Educational, Scientific and Cultural Organization Friederike (UNESCO), in: Rüdiger Wolfrum (Hrsg.), Max Planck Encyclopedia of Public International Law (MPEPIL), August 2010.

Benatar, David Bioethics and Health and Human Rights: A Critical View, 32 Journal of Medical Ethics 1 (2006) 17 ff.

Clados, Miriam Chances for and Limits of International Law and Legal Language in the Area of Bioethics, in: Silja Vöneky, Cornelia Hagedorn, Miriam Clados, Jelena von Achenbach (Hrsg.), Legitimation ethischer Entscheidungen im Recht. Interdisziplinäre Untersuchungen, Springer, Berlin/Heidelberg 2009, 297 ff.

Duttge, Gunnar „Was meint genetische Individualität?“ - Eine Problemskizze, in: Gunnar Duttge, Wolfgang Engel, Barbara Zoll (Hrsg.), Genetische Individualität im Recht, Göttinger Schriften zum Medizinrecht, Bd. 
13, Universitätsverlag Göttingen, Göttingen 2012, 3 ff.

Farmer, Paul/

Campos Gastineau, Journal of Law, Medicine and Ethics 2 (2004) $243 \mathrm{ff}$.

Nicole

Forschner,

Maximilian

Friedrich, Jürgen

Friele, Minou

Fuhrmann, Manfred

Stierle (Hrsg.), Identität. Poetik und Hermeneutik, Bd. VIII, Wilhelm Fink, München 2. Aufl. 1996, 83 ff.

Gadzicki, Dorothea

Der Stand des genetischen Möglichen und die Rolle der genetischen Beratung - jetzt und in Zukunft, in: Thorsten Moos, Jörg Niewöhner, Klaus Tanner (Hrsg.), Genetisches Wissen, Röhrig Universitätsverlag, St. Ingebert 2011, 49 ff.

Gros Espiell, Héctor The Preamble, in: Henk ten Have, Michèle Stanton-Jean (Hrsg.), The UNESCO Universal Declaration on Bioethics and Human Rights. Background, principles and application, UNESCO, Paris $2009,57 \mathrm{ff}$.

Habermas, Jürgen
Die Zukunft der menschlichen Natur. Auf dem Weg zu einer 
liberalen Eugenik? Suhrkamp, Frankfurt am Main 2001.

Höfling,Wolfram Definiert die Rechtsordnung den Menschen? In: Walter Schweidler (Hrsg.), Menschenleben - Menschenwürde. Internationales Symposium zur Bioethik, Lit Verlag, Münster/Hamburg/London 2003, $165 \mathrm{ff}$.

Hofmann, Hasso Biotechnik, Gentherapie, Genmanipulation - Wissenschaft im rechtsfreien Raum? JuristenZeitung 6 (1986) 253 ff.

Jacqué, Jean-Paul Europäische Menschenrechtskonvention und Bioethik, in: Françoise Furkel, Heike Jung (Hrsg.), Bioethik und Menschenrechte/Bioéthique et droits de l'homme, Heymann, Köln u. a. 1993, $1 \mathrm{ff}$.

Kant, Immanuel Grundlegung zur Metaphysik der Sitten, Kommentar von C. Horn/C. Mieth/N. Scarano, Suhrkamp, Frankfurt am Main 2007 (AA IV).

Kather, Regine

Person. Die Begründung menschlicher Identität, Wissenschaftliche Buchgesellschaft, Darmstadt 2007.

Kirchhof, Paul Menschenbild und Freiheitsrecht, in: Rainer Grote, Ines Härtel, Karl-Eberhard Hein, Thorsten Ingo Schmidt, Thomas Schmitz, Gunnar Folke Schuppert, Christian Winterhoff (Hrsg.), Die Ordnung der Freiheit. FS für Christian Starck zum 70. Geburtstag, Mohr Siebeck, Tübingen 2007, 275 ff.

Klein, Eckart Impact of Treaty Bodies on the International Legal Order, in: Rüdiger Wolfrum, Volker Röben (Hrsg.), Developments of International Law in Treaty Making, Springer, Berlin/Heidelberg $2005,571 \mathrm{ff}$.

Kobusch, Theo

Die Entdeckung der Person. Metaphysik der Freiheit und modernes Menschenbild, Herder, Freiburg, 1993.

Kollek, Reginel Der medizinische Blick in die Zukunft. Gesellschaftliche Lemke, Thomas Implikationen prädiktiver Gentests, Campus, Frankfurt am 
Main/New York 2008.

Langlois, Adèle $\quad$ Negotiating Bioethics, Routledge, London 2013.

Liao, S Matthew Is there a duty to share genetic information? 35 Journal of Medical Ethics 4 (2009) 306 ff.

Mahlmann,

Matthias

McCrudden,

Christopher

Mieth, Dietmar/

Düwell, Marcus

Molnár-Gábor,

Fruzsina/ Gantner,

Gösta/ Lichter,

Peter

Molnár-Gábor,

Fruzsina/ Weiland,

Johannes

Muñoz Hernandez, Fernando

Ohlin, Jens David

Porz, Rouven
Elemente einer ethischen Grundrechtstheorie, Nomos, Baden-Baden 2008.

Human Dignity and Judicial Interpretation of Human Rights, 19 European Journal of International Law 4 (2008) 655 ff.

Ethische Überlegungen zur UNESCO-Deklaration über das menschliche Genom und die Menschenrechte, in: Dietmar Mieth (Hrsg.), Was wollen wir können? Ethik im Zeitalter der Biotechnik, Herder, Freiburg/Basel/Wien 2002, 344 ff.

Das Ende der Zufallsbefunde in der genetischen Diagnostik. Die Ganzgenomsequenzierung und die Erwartbarkeit zusätzlicher Befunde, Zeitschrift für Medizin-Ethik-Recht 2 (2014) $81 \mathrm{ff}$.

Die Totalsequenzierung des menschlichen Genoms als medizinischer Eingriff - Bewertung und Konsequenzen, 60 Zeitschrift für medizinische Ethik 2 (2014) 135 ff.

Legitimität und Legalität im Völkerrecht. Die völkerrechtstheoretische Debatte um die Legitimität GovernanceStrukturen, Inaugural-Dissertation Universität Tübingen, 2011.

Is the concept of the person necessary for human rights? 105 Columbia Law Review 1 (2005) 209 ff.

The Need for an Ethics of Kinship: Decision Stories and Patients's Context, in: Christoph Rehmann-Sutter, Hansjakob Müller (Hrsg.), Disclosure Dilemmas. Ethics of Genetic Prognosis after the 'Right to know/Not to Know' Debate, Ashgate, Farnhma/Burlington 2009, 53 
Rehmann-Sutter, Communication Yes, but How - and What? In: Christoph RehmannChristoph Sutter, Hansjakob Müller (Hrsg.), Disclosure Dilemmas. Ethics of Genetic Prognosis after the 'Right to know/Not to Know' Debate, Ashgate, Farnhma/Burlington 2009, 45 ff.

Reichold, Anne

Die vergessene Leiblichkeit. Zur Rolle des Körpers in ontologischen und ethischen Personentheorien, Mentis, Paderborn 2004.

Sandkühler, Hans Enzyklopädie Philosophie, Bd. 2, Felix Meiner Verlag, Hamburg Jörg (Hrsg.) 2010.

Schmidt, Markus G. Reform of the United Nations Human Rights Programs - current challenges and trends, in: Helmut Volger, Norman Weiß (Hrsg.), Die Vereinten Nationen vor globalen Herausforderungen, Referate der Potsdamer UNO-Konferenzen 2000-2008, Universitätsverlag, Potsdam 2011, $81 \mathrm{ff}$.

Stokke, Olav S. Regimes as Governance Systems, in: Oran R. Young (Hrsg.), Global Governance: drawing insights from the environmental experience, MIT Press, Cambridge, MA u.a. 1997, 27 ff.

Schücklenk, Udo/ International Research Ethics, 14 Bioethics 2 (2000) $158 \mathrm{ff}$.

Ashcroft, Richard

Sturma, Dieter

Person, in: Hans Jörg Sandkühler (Hrsg.), Enzyklopädie Philosophie, Bd. 2, Felix Meiner Verlag, Hamburg 2010, 1921 ff.

Sturma, Dieter

Person, in: Petra Kolmer, Armin Wildfeuer (Hrsg.), Neues Handbuch philosophischer Grundbegriffe, Bd. II, Verlag Karl Alber, Freiburg im Breisgau 2011, 1728 ff.

Taylor, Allyn L. Globalization and biotechnology: UNESCO and an International Strategy to Advance Human Rights and Public Health, 25 American Journal of Law and Medicine 4 (1999) 479 ff.

ten Have, Henk Kulturübergreifende Bioethik. Der Ansatz der UNESCO, in: Ludger 
Honnefelder, Dirk Lanzerath (Hrsg.), Bioethik im Kontext von Recht, Moral und Kultur. Beiträge der Internationalen Konferenz vom 14.-15.12.2005 in Berlin, University Press, Bonn 2008, 123 ff.

ten Have, Henk/

Stanton-Jean,

Michèle

Thumfart,

Alexander

Thürer, Daniel

Tymstra, Tjeerd

The UNESCO Universal Declaration on Bioethics and Human Rights. Background, principles and application, UNESCO, Paris 2009.

Die Würde des Menschen: Giovanni Pico della Mirandola, Albrecht Dürer und Avishai Margalit, 51 Zeitschrift für Politik 4 (2004) 434 ff.

Soft law, in: Wolfrum, Rüdiger (Hrsg.), Max Planck Encyclopedia of Public International Law (MPEPIL), März 2009.

'If I Only Had (Not) Known That': About Risk-Individualization, Chnce Specification and the Loss of Certainty of Not-Knowing, in: Christoph Rehmann-Sutter, Hansjakob Müller (Hrsg.), Disclosure Dilemmas. Ethics of Genetic Prognosis after the 'Right to know/Not to Know' Debate, Ashgate, Farnhma/Burlington 2009, 85 ff.

Van El, Carla G/ Whole-genome sequencing in health care, 21 European Journal of Cornel, Martina C/ Human Genetics 6 (2013) 580 ff.

Borry, Pascal/

Hastings, Ros J/

Fellmann, Florencel

Hodgson, Shirley VI

Howard, Heidi C/

Cambon-Thomson,

Annel Knoppers,

Bartha Maria,

Meijers-Heijboer,

Hanne/ Scheffer,

Hans/Tranebjaerg,

Lisbeth/ Dondorp,

Wybo/ de Wert, 
Guido MWR

Vöneky, Silja

Ethische Standards im Wissenschaftsrecht, in: Wolfgang Löwer, Klaus Ferdinand Gärditz (Hrsg.), Wissenschaft und Ethik, Wissenschaftsrecht: Zeitschrift für deutsches und europäisches Wissenschaftsrecht, Beiheft 21/2012, 68 ff.

Vöneky, Silja

Grundlagen und Grenzen der Ethisierung des Völkerrechts, in: Silja Vöneky, Britta Beylage-Haarmann, Anja Höfelmeier, AnnaKatharina Hübler (Hrsg.), Ethik und Recht - Die Ethisierung des Rechts, Springer, Heidelberg u.a. 2013, 129 ff.

Vöneky, Silja/ Legitimation ethischer Entscheidungen im Recht. Interdisziplinäre Hagedorn, Untersuchungen, Springer, Berlin/Heidelberg 2009.

Cornelia/ Clados,

Miriam/von

Achenbach, Jelena

(Hrsg.)

Winkler, David/ Prevention of Harmful False Diagnosis versus Threat by Disclosure Lyrer, Philippe $\quad$ of an Inheritable Disease - A Medical Case, in: Christoph RehmannSutter, Hansjakob Müller (Hrsg.), Disclosure Dilemmas. Ethics of Genetic Prognosis after the 'Right to know/Not to Know' Debate, Ashgate, Farnhma/Burlington 2009, 39 ff.

Wolfrum, Rüdiger Legitimacy in International Law, in: Rüdiger Wolfrum (Hrsg.), Max Planck Encyclopedia of Public International Law (MPEPIL), März 2011.

Wolfrum, Rüdiger Samuel Pufendorfs Blick auf die EU. Eine Hypothese, in: Christian Baldus, Herbert Kronke, Ute Mager (Hrsg.), Heidelberger Thesen zu Recht und Gerechtigkeit, Mohr Siebeck, Tübingen 2013, 27 ff. 